\title{
Amplitude of the Earth's Free Oscillations and Long-Period Characteristics of the Earthquake Source
}

\author{
Hiroo Kanamori and Don L. Anderson \\ Seismological Laboratory, California Institute of Technology \\ Pasadena, California 91109
}

\begin{abstract}
Amplitude spectra of the spheroidal modes ${ }_{0} S_{l}(l=2-40)$ excited by the 1960 Chilean earthquake and observed at Pasadena, Los Angeles, and Isabella show distinct holes at ${ }_{0} S_{10}(T=580 \mathrm{~s})$ and ${ }_{0} S_{21}(T=336$ s). These holes can be explained as an interference pattern caused by a composite source consisting of a finite propagating source (main shock) and a slow precursory source, $15 \mathrm{~min}$ before the main shock. A total seismic moment of $4-5 \times 10^{30}$ dyn $\mathrm{cm}$ is required to explain the observed amplitude.
\end{abstract}

The eigenperiods and attenuation of the earth's free oscillations have been extensively studied in recent years. However, the amplitude of the earth's free oscillations has received relatively little attention. Since the amplitude spectrum depends on the spacio-temporal characteristics of the source, it provides important information concerning the eafthquake source dynamics. Earlier attempts to utilize free oscillation amplitude for source studies include Kovach and Anderson [1967] and $W u$ [1966]. Abe [1970] made a detailed analysis of the free oscillations excited by the Kurile Islands earthquake of $1963\left(M_{s}=8.3\right)$ and concluded that the seismic moment determined by the free oscillation data up to $600 \mathrm{~s}$ is consistent with that determined by shorter-period surface wave data. This conclusion suggests a more or less step function source dislocation. Ben-Menahem et al. [1972] arrived at a similar conclusion. Gilbert [1970] and Dziewonski and Gilbert [1974] introduced a moment tensor representation of the earthquake source to analyze normal mode spectra systematically. Dziewonski and Gilbert [1974] applied it to two deep focus earthquakes and suggested a precursory isotropic deformation at the source.

The Chilean earthquake of $1960\left(M_{s}=8.3\right)$, one of the largest events ever recorded, excited a large number of torsional and spheroidal modes including the gravest mode [e.g., Benioff et al., 1961; Ness et al., 1961; Alsop et al., 1961]. The amplitude spectrum presented by these authors has a distinct structure (see Figures 1 and 3); for example, the spectrum of a gravity record obtained at the University of California at Los Angeles (UCLA) shows distinct spectral holes at ${ }_{0} S_{10}(T=9.65$ $\min )$ and ${ }_{0} S_{21}(T=5.6 \mathrm{~min})$. It is not obvious that this structure is compatible with the ordinary earthquake source dislocation, a step function in time as envisaged in the previous studies. In fact, Alsop [1964] pointed out a difficulty in explaining the amplitude in terms of a simple source model. Kanamori and Cipar [1975] (hereafter abbreviated to paper 1) suggest, on the basis of long-period surface waves and body waves, that the Chilean earthquake is larger, by far, than any earthquake ever recorded and that it was preceded by a slow aseismic slip of about the same magnitude as the main shock. This precursor has a time constant of $300-600 \mathrm{~s}$ and started about $1000 \mathrm{~s}$ before the main shock. This conclusion is based on a single seismogram recorded at Pasadena and needs further verification. The present paper represents an attempt to use the spectral amplitude data for investigating such complex source characteristics of large earthquakes.

Copyright $\odot 1975$ by the American Geophysical Union.

\section{DATA}

The basic data sets used here are the UCLA gravity records [Ness et al., 1961], Pasadena Press-Ewing records, and Isabella strain records [Benioff et al., 1961; Smith, 1961]. We reanalyzed the raw data by direct Fourier analysis to obtain the harmonic amplitudes of the spheroidal oscillations. The overall shape of the spectrum obtained by this new analysis is almost identical to the overall shapes given by Ness et al. [1961] and Benioff et al. [1961]. The details of the data are summarized in Table 1. For the Pasadena Press-Ewing and the Isabella strain records, corrections are made for the instrument response, which was calibrated by a method similar to that described in paper 1. For the UCLA gravity record the harmonic amplitudes of gravity for all the modes except ${ }_{0} S_{2}(T=54 \mathrm{~min})$ are multiplied by $T^{2} / 4 \pi^{2}$ to obtain the harmonic amplitudes of displacement. For the ${ }_{0} S_{2}$ mode the 'free air' and the 'Bouguer' terms become comparable to the acceleration term $T^{2} / 4 \pi^{2}$ and are included in converting gravity to displacement. Since the analysis is made for a record section that starts at $t_{0}$ and ends at $t_{0}+\tau$ after the origin time of the earthquake, the amplitudes are multiplied by a factor

$$
\frac{\pi \tau / Q T}{[1-\exp (-\pi \tau / Q T)] \exp \left(-\pi t_{0} / Q T\right)}
$$

to correct for the attenuation of the free oscillations. For the values of $Q$ factors a smooth curve is fitted to the data points of Figure 7 of Smith [1972], and the values were read from this curve. Table 2 lists the $Q$ values actually used. The resulting amplitude can be regarded as the amplitude of the harmonic component of the free oscillations in a nonattenuating earth. In Figures 1, 2, 3, and 4 the amplitude of each mode is plotted. One problem arises for the UCLA gravity record. Since the Pasadena Press-Ewing and the UCLA gravity records represent the vertical component of the spheroidal oscillations at nearby locations, they should agree with each other. However, the amplitudes determined by the UCLA gravity record are nearly $1 / 30$ of those determined by the Pasadena Press-Ewing record. This difference is suspected to be a result of a sluggish servo-nulling system of the UCLA gravity meter; the servo system could not follow the very rapid change in the gravity caused by the free oscillations (J. C. Harrison, private communication, 1974). Thus the absolute level of the amplitude determined by the UCLA gravity record is not reliable. However, it is unlikely that this sluggish response affects the shape of the amplitude spectrum. The amplitudes of the UCLA gravity record are arbitrarily multiplied by 28 and 


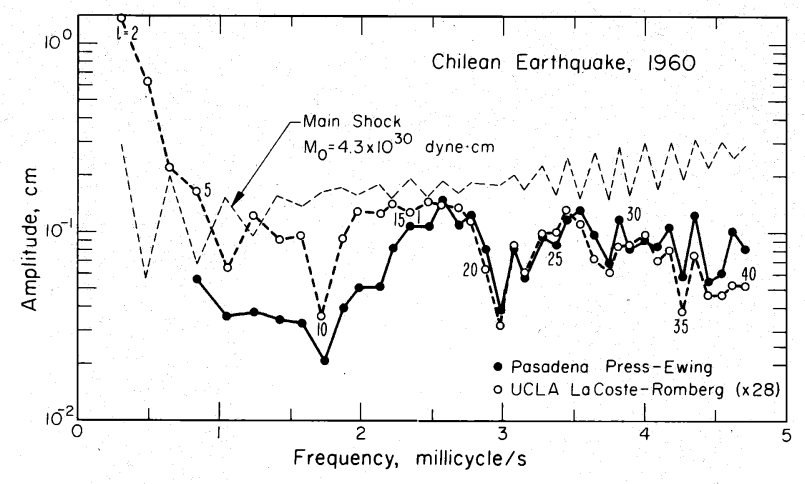

Fig. 1. The amplitude of the spheroidal modes $(l=2-40)$ determined by spectral analysis of the Pasadena Press-Ewing record (closed circles) and the UCLA gravity record (open circles) of the 1960 Chilean earthquake. The points for each mode are connected to show the general trend. The dashed curve shows the amplitude calculated for a finite moving source. The seismic moment is arbitrarily set at 4.3 $\times 10^{30}$ dyn $\mathrm{cm}$.

plotted in Figures 1 and 2. As is shown in Figure 1, the shape of the spectrum of the UCLA gravity record is very similar to that of the Pasadena Press-Ewing record, the suggestion being that the spectral holes at $S_{10}$ and $S_{01}$ are real. A similar trend is seen in the Isabella strain spectrum (Figure 3).

\section{INTERPRETATION}

In general, spectral holes suggest some sort of interference. If two excitations of about the same intensity are given, with a time separation of $\Delta \tau$, to a linear system, the response of the system will have holes at periods $T_{1}=2 \Delta \tau, T_{2}=2 \Delta \tau / 3, T_{3}=$ $2 \Delta \tau / 5$, and $T_{4}=2 \Delta \tau / 7$. The precursor model of the Chilean earthquake proposed in paper 1 suggests $\Delta \tau=15 \mathrm{~min}=900 \mathrm{~s}$, which gives $T_{1}=1800 \mathrm{~s}, T_{2}=600 \mathrm{~s}, T_{3}=360 \mathrm{~s}, T_{4}=257 \mathrm{~s}$, $\ldots$. The periods of $S_{0} S_{10}$ and $S_{01}, 580$ and $336 \mathrm{~s}$, respectively, are close to $T_{2}$ and $T_{3}$; thus the observed holes seem consistent with the proposed precursory model. Of course, the actual problem is more complicated because of the finite source geometry and finite time constant of the precursor, which make the periods of such spectral holes azimuthally dependent. However, the above comparison is encouraging enough to warrant the more detailed analysis that follows.

The $l$ th mode of the free oscillation at $\mathbf{r}$ excited by a point double couple at $r_{0}$, varying stepwise in time, is given by

$$
U_{l}=M_{0} C_{l}\left(\mathrm{r}-\mathrm{r}_{0}\right) \cos \sigma_{l} t
$$

where $M_{0}$ is the seismic moment, $\sigma_{l}$ is the angular eigen-

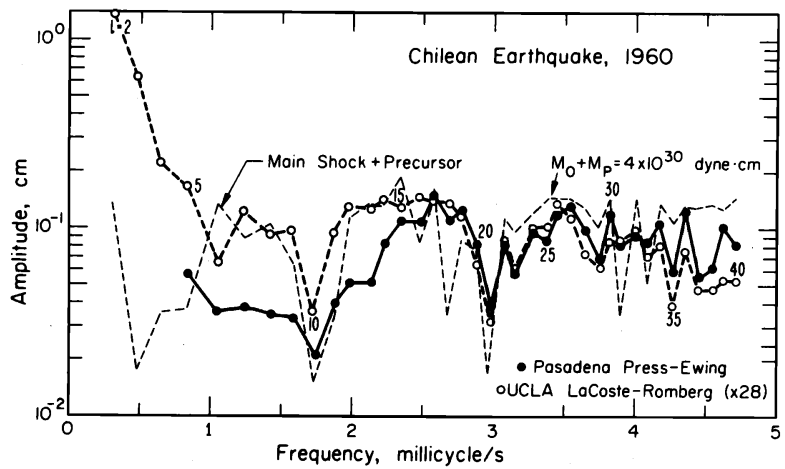

Fig. 2. The amplitude of the spheroidal modes $(l=2-40)$. The dashed curve is calculated for a composite source consisting of a finite moving source and a long-period precursor.

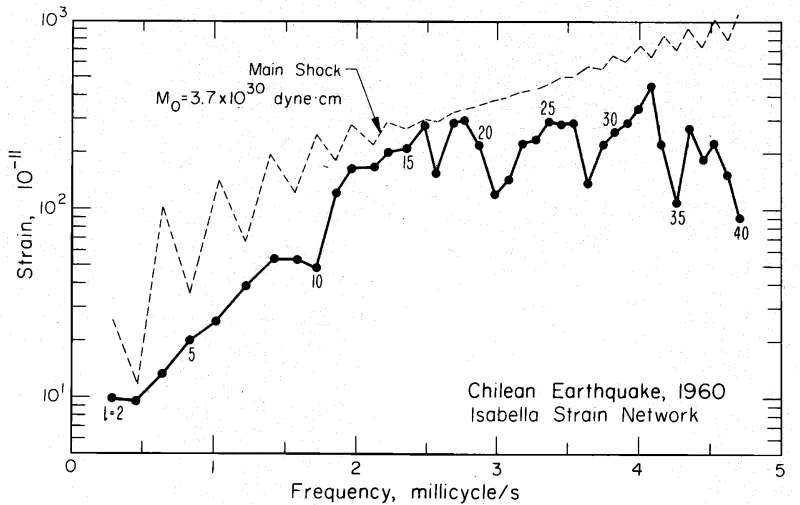

Fig. 3. Strain amplitudes of the spheroidal oscillations $(l=2-40)$ excited by the 1960 Chilean earthquake. The dashed curve shows the amplitude for a finite moving source. The moment is arbitrary.

frequency, and $C_{l}$ is the amplitude factor, which can be computed from the eigenfunctions for any source geometry [Saito, 1967; Ben-Menahem et al., 1971; Gilbert, 1970; Kanamori and Cipar, 1975]. At periods longer than $1000 \mathrm{~s}$ the effect of the rotational splitting becomes significant. However, since the actual calculations are too involved to be performed at the present time, it is ignored here.

A finite propagating source can be approximated by a finite number $N$ of point sources aligned at $\Delta L=L / N$ intervals, where $L$ is the 'fault' length. Each point source is imparted a moment $M_{0} / N$ and is turned on, successively, at $\Delta t=\Delta L / v$ intervals, where $v$ is the rupture velocity. The displacement is then given by

$U_{l}=\frac{M_{0}}{N} \sum_{j=1}^{N} C_{l}\left(\mathrm{r}-\left(\mathrm{r}_{0}+j \Delta L 1\right)\right) \cos \sigma_{l}(t-j \Delta L / v)$

where 1 is the unit vector in the direction of the propagation. Writing $C_{l}\left(\mathrm{r}-\left(\mathrm{r}_{0}+j \Delta L \mathrm{l}\right)\right)=C_{l j}$, we have

$$
U_{l}=M_{0} \bar{C}_{l} \cos \left(\sigma_{l} t-\phi_{l}\right)
$$

where

$$
\begin{aligned}
\bar{C}_{l}=\frac{1}{N}\left[\left(\sum_{j=1}^{N} C_{l j} \cos \right.\right. & \left.\frac{\sigma_{l} j \Delta L}{v}\right)^{2} \\
& \left.+\left(\sum_{j=1}^{N} C_{l j} \sin \frac{\sigma_{l} j \Delta L}{v}\right)^{2}\right]^{1 / 2}
\end{aligned}
$$

and

$$
\begin{aligned}
\phi_{l}= & \tan ^{-1} \\
& \cdot\left(\sum_{j=1}^{N} C_{l j} \sin \sigma_{l} j \Delta L / v\right) /\left(\sum_{j=1}^{N} C_{l j} \cos \sigma_{l} j \Delta L / v\right)
\end{aligned}
$$

Thus the harmonic amplitudes for this source are given by $M_{0} \bar{C}_{l}$.

In Figure $1, M_{0} \bar{C}_{l}$ computed for the source geometry given by paper 1 are plotted for $l=2-40$. The source parameters are depth, $53 \mathrm{~km}$; fault strike, $\mathrm{N} 10^{\circ} \mathrm{E}$; dip angle, $10^{\circ}$; and slip angle, $90^{\circ}$. The rupture starts at $38^{\circ} \mathrm{S}$ and $73.5^{\circ} \mathrm{W}$ and propagates in a $\mathrm{S} 10^{\circ} \mathrm{W}$ direction over a distance of $800 \mathrm{~km}$ with a rupture velocity of $3.5 \mathrm{~km} / \mathrm{s}$. Numerical calculation is made with $\Delta L=50 \mathrm{~km}$ and $N=16$. Although the spectrum is rather jagged for a point source (not shown), it is averaged out for the finite source and becomes nearly flat over the entire frequency range. The value of $M_{0}$ simply sets the level of the curve. Here $M_{0}=4.3 \times 10^{30} \mathrm{dyn} \mathrm{cm}$ is used to avoid cluttering the figure. The average amplitude of the observed Press-Ewing 
TABLE 1. Starting Time $\left(t_{0}\right)$, Duration $(\tau)$, and Sampling Interval $(\Delta t)$ of the Records

\begin{tabular}{lcccc}
\hline & $t_{0}, \mathrm{~s}$ & $\tau, \mathrm{s}$ & $\Delta i, \mathrm{~s}$ & Mode \\
\hline $\begin{array}{l}\text { Pasadena Press-Ewing } \\
\text { (vertical) }\end{array}$ & 24,600 & 40,960 & 10 & ${ }_{0} S_{5} \sim{ }_{0} S_{40}$ \\
UCLA gravity & 22,920 & 245,760 & 60 & ${ }_{0} S_{2} \sim{ }_{0} S_{12}$ \\
Isabella strain & 22,920 & 122,880 & 60 & ${ }_{0} S_{13} \sim{ }_{0} S_{40}$ \\
& 17,340 & 245,760 & 60 & ${ }_{0} S_{2} \sim{ }_{0} S_{12}$ \\
& 17,340 & 122,880 & 60 & ${ }_{0} S_{13} \sim{ }_{0} S_{40}$ \\
\hline
\end{tabular}

spectrum indicates $M_{0} \sim 2 \times 10^{30}$ dyn $\mathrm{cm}$, which agrees reasonably well with that given by paper 1 . It is evident that the prominent holes at $S_{10}$ and $S_{21}$ cannot be explained in terms of this finite source alone. Now we introduce a precursory source. The precursory source proposed by paper 1 has the same mechanism as the main shock and is given by a dislocation function

$$
\begin{array}{rrrl}
D(t) & =0 & t & <-t_{p} \\
D(t) & =\frac{1}{2}\left(1-\cos \frac{\pi\left(t+t_{p}\right)}{\tau_{0}}\right) & -t_{p} & \leq t<-t_{p}+\tau_{0} \\
D(t) & =1 & t & \geq-t_{p}+\tau_{0}
\end{array}
$$

where $-t_{p}$ is the origin time of the precursor with respect to the main shock and $\tau_{0}$ is the time constant of the precursor. Since the spectrum of this function is

$$
\hat{D}(\sigma)=\frac{\pi^{2} \cos \frac{1}{2} \sigma \tau_{0}}{i \sigma\left(\pi^{2}-\sigma^{2} \tau_{0}^{2}\right)} \exp \left[i \sigma\left(t_{p}-\tau_{0} / 2\right)\right]
$$

The $l$ th mode caused by this precursor becomes

$$
M_{p} \tilde{C}_{l} \cos \left(\sigma_{l}\left(t+t_{p}-\tau_{0} / 2\right)\right)
$$

where

$$
\tilde{C}_{l}=C_{l} \pi^{2} \cos 1 / 2 \sigma_{l} \tau_{0} /\left(\pi^{2}-\sigma_{l}{ }^{2} \tau_{0}{ }^{2}\right)
$$

and $M_{p}$ is the seismic moment of the precursor. Since the finiteness effect is not important for a slow precursory motion, here we represent it by a point source placed at $41.5^{\circ} \mathrm{S}$ and $74.3^{\circ} \mathrm{W}$, near the center of the aftershock area. This precursor together with the main shock gives the resultant $l$ th mode:

$$
\begin{gathered}
M_{0} \bar{C}_{l} \cos \left(\sigma_{l} t-\phi_{l}\right)+M_{P} \tilde{C}_{l} \cos \left(\sigma_{l}\left(t+t_{p}-1 / 2 \tau_{0}\right)\right) \\
=\left(M_{0}+M_{p}\right) C_{l}{ }^{*} \cos \left(\sigma_{l} t-\xi_{l}\right) \\
C_{l}{ }^{*}=\left(M_{0}+M_{p}\right)^{-1}\left[\left(M_{0} \bar{C}_{l}\right)^{2}+\left(M_{p} \tilde{C}_{l}\right)^{2}\right. \\
\left.+2 M_{0} M_{p} \bar{C}_{l} \tilde{C}_{l} \cos \left(\phi_{l}+\sigma_{l} t_{p}-1 / 2 \sigma_{l} \tau_{0}\right)\right]^{1 / 2} \\
\xi_{l}=\tan ^{-1} \frac{M_{0} \bar{C}_{l} \sin \phi_{l}+M_{p} \tilde{C}_{l} \sin \left(-\sigma_{l} t_{p}+\frac{1}{2} \sigma_{l} \tau_{0}\right)}{M_{0} \bar{C}_{l} \cos \phi_{l}+M_{p} \tilde{C}_{l} \cos \left(-\sigma_{l} t_{p}+\frac{1}{2} \sigma_{l} \tau_{0}\right)}(8)
\end{gathered}
$$

Actually, $\mathcal{C}_{l}, \tilde{C}_{l}$, and $\phi_{l}$ are complicated functions of source geometry, but they can be computed in a straightforward manner by (4), (5), and (6). We computed (7) for various combinations of $t_{p}$ and $\tau_{0}$ and for $M_{0}=M_{p}$ as suggested by paper 1 ; we found that the results for $t_{p}=900 \mathrm{~s}$ and $\tau_{0}=300 \mathrm{~s}$ can explain the holes at ${ }_{0} S_{10}$ and $S_{21}$ as shown in Figure 2. These values are consistent with those obtained by Kanamori and Cipar [1975] from the direct precursor signal on the Pasadena strain seismogram. The resultant moment $M_{0}+M_{p}$ is estimated as $4 \times 10^{30}$ dyn $\mathrm{cm}$ (Figure 2), which agrees reasonably well with that given by paper 1 .

A similar analysis is made for the strain record. Although the expressions corresponding to $C_{l}$ are somewhat more complicated, the calculation is straightforward. Figures 3 and 4 show the harmonic amplitude of the strain for the finite main shock and precursor plus main shock, respectively. Although the strain spectrum is more complicated than the displacement spectrum, probably because of the coupling between torsional and spheroidal modes (e.g., ${ }_{0} T_{7-0} S_{7},{ }_{0} T_{11-0} S_{10}$, and $S_{01{ }^{-0}} T_{12}$ ), the agreement between the observed and calculated spectra for the precursor plus main shock seems better than that for the main shock alone. A total moment of $5 \times 10^{30}$ dyn $\mathrm{cm}$ is suggested (Figure 4).

\section{Discussion AND Conclusions}

The present analysis shows that the spectral holes can be interpreted as an interference pattern caused by the main shock and the precursor. It should be mentioned, however, that since we did not use the phase spectrum, which is much more difficult to retrieve, it is not possible, from the present analysis alone, to tell whether the interfering event is precursory or postseismic. The precursory signal on the Pasadena strain record reported in paper 1 together with the spectral holes, however, leads us to conclude that a significant precursory displacement did occur at least 15 min before the 1960 Chilean earthquake. If the precursory signal is a result of instrumental instability or nonlinearity, though this is very unlikely, then the interfering event represents a postseismic deformation.

The amplitude spectrum observed at other stations [e.g., Alsop et al., 1961; Alsop, 1964; Dziewonski and Landisman, 1970; Bolt and Marussi, 1961] shows spectral holes similar to those reported here, although some of them show rather complicated patterns. For example, spectral holes near ${ }_{0} S_{10}$ are found as follows: Resolute, Lwiro, ${ }_{0} S_{10}$; Suva, Uppsala, Palisade, ${ }_{0} S_{9}$ (633 s); Cape Hallett, Hong Kong, Mt. Tsukuba, ${ }_{0} S_{12}(501 \mathrm{~s})$; and Arga, ${ }_{0} S_{11}(536 \mathrm{~s})$. Spectral holes near $S_{21}$ are Suva, Uppsala, ${ }_{0} S_{23}(315 \mathrm{~s})$; Cape Hallett, ${ }_{0} S_{22}(325 \mathrm{~s})$; Resolute, ${ }_{0} S_{20}(347 \mathrm{~s})$; Arga, ${ }_{0} S_{19}(360 \mathrm{~s})$; Mt. Tsukuba, ${ }_{0} S_{18}$ (374 s); and Hong Kong, Matsuhiro, ${ }_{0} S_{17}(390 \mathrm{~s})$. Some of these holes are not very pronounced. Also spectral holes are found at other periods. Because of the directivity of the main shock and, possibly, of the precursor the periods where the destructive interference takes place are azimuthally dependent. Therefore the position and the degree of the spectral holes can

TABLE 2. $Q$ of ${ }_{0} S_{l}$

\begin{tabular}{llll}
\hline$l$ & $Q$ & $l$ & $Q$ \\
\hline 2 & 480 & 22 & 206 \\
3 & 440 & 23 & 202 \\
4 & 410 & 24 & 198 \\
5 & 390 & 25 & 194 \\
6 & 370 & 26 & 191 \\
7 & 350 & 27 & 188 \\
8 & 330 & 28 & 185 \\
9 & 315 & 29 & 182 \\
10 & 300 & 30 & 180 \\
11 & 285 & 31 & 178 \\
12 & 270 & 32 & 176 \\
13 & 260 & 33 & 174 \\
14 & 250 & 34 & 172 \\
15 & 240 & 35 & 170 \\
16 & 230 & 36 & 168 \\
17 & 225 & 37 & 166 \\
18 & 220 & 38 & 164 \\
19 & 217 & 39 & 162 \\
20 & 214 & 40 & 160 \\
21 & 210 & & \\
& & &
\end{tabular}




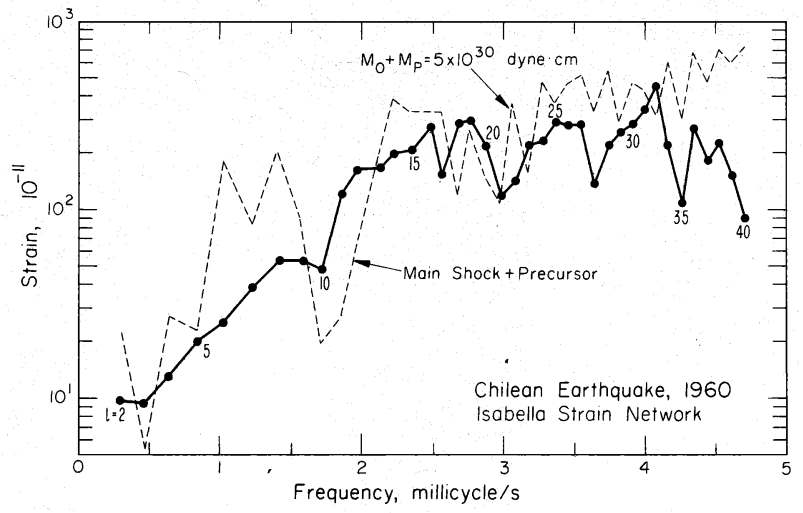

Fig. 4. The strain amplitude of the spheroidal oscillations $(l=$ 2-40). The dashed curve is calculated for a composite source consisting of a finite moving source and a long-period precursor.

be considerably different from station to station. When the periods of the destructive interference fall between the consecutive eigenperiods, pronounced spectral holes can no longer be expected. At a certain distance the intrinsic modal pattern of the free oscillations causes peaks and troughs in the spectral amplitude. A calculation carried out for Trieste shows that such a pattern does indeed exist. The torsional spectrum calculated for the proposed precursor plus main shock shows no pronounced holes other than those corresponding to the intrinsic free oscillation modal patterns. It is obviously important to test the present model in more detail against these free oscillation data obtained at other stations. However, most of these records are obtained by 15-100 long-period seismographs, and the most important spectral data of lowerorder $(l<10)$ oscillations are not very reliable. In view of this unreliability and the unavailability, at hand, of raw data at these stations with absolute amplitude scale, we did not carry out such analysis in the present paper.

Figures 2 and 4 show significant disagreement between the observed and calculated spectra for $l<5$ of the displacement spectrum (Figure 2) and for $l<8$ of the strain spectrum. It is quite possible that the source time history may have an even longer period component than that postulated here. If the upgoing trend of the observed gravity spectrum toward low order (see Figure 2) is a real feature, it indicates a long-period source dislocation that is nearly 1 order of magnitude larger than that postulated here. This large long-period source dislocation is an interesting possibility in relation to the effect of earthquakes on global problems of the earth, such as the translational oscillation of the inner core [Slichter, 1961], plate motions, the earth's polar motion, and the change in the earth's rotation rate. However, as was mentioned earlier, the gravity data are subject to large uncertainty because of the sluggish, probably nonlinear, response of the servo system. Since the effect of the sluggishness could be less at a 1 -hr period $\left(\sim_{0} S_{2}\right)$ than at shorter periods, the multiplication of the entire spectrum by a constant factor (28) might have overestimated the spectral height at the long-period end. Also no such trend is found for the strain spectrum; for $l<5$ the observed trend is more or less consistent with the calculated trend. However, at long periods the strain becomes very small, and the signal-to-noise ratio becomes poor. The above agreement may be only fortuitous. Confirmation of the possibility of such large-scale long-period motion must await more reliable free oscillation spectra.
The present results are admittedly based on relatively small numbers of data sets. We therefore do not claim uniqueness for the solution but would like to emphasize that the Chilean earthquake, a truly significant event in this century, has a very long period displacement component, which cannot be explained by an ordinary step function dislocation model of earthquakes. Such a component suggests that earthquakes may represent a more pronounced tectonic process than they were heretofore considered.

Acknowledgments. We thank Louis B. Slichter and Stewart W. Smith for kindly providing us with the digitized records of the Chilean earthquake. Christopher Harrison, Francis Lehner, and Len Blayney provided us with useful information for the calibration of the instruments. We also thank the reviewers of this paper for constructive comments. This work was supported by National Science Foundation grant GA 40752 and Advanced Research Projects Agency grant F44620-72-C-0078. Contribution 2521, Division of Geological and Planetary Sciences, California Institute of Technology.

\section{REFERENCES}

Abe, K., Determination of seismic moment and energy from the earth's free oscillation, Phys. Earth Planet. Interiors, 4, 49-61, 1970.

Alsop, L. E., Spheroidal free periods of the earth observed at eight stations around the world, Bull. Seismol. Soc. Amer., 54, 755-776, 1964.

Alsop, L. E., G. H. Sutton, and M. Ewing, Free oscillations of the earth observed on strain and pendulum seismographs, J. Geophys. Res., 66, 631-641, 1961.

Ben-Menahem, A., M. Israel, and U. Levité, Theory and computation of amplitudes of terrestrial line spectra, Geophys. J., 25, 309-408, 1971.

Ben-Menahem, A., M. Rosenman, and M. Israel, Source mechanism of the Alaskan earthquake of 1964 from amplitudes of free oscillations and surface waves, Phys. Earth Planet. Interiors, 5, 1-29, 1972.

Benioff, H., F. Press, and S. Smith, Excitation of the free oscillations of the earth by earthquakes, J. Geophys. Res., 66, 605-619, 1961.

Bolt, B. A., and A. Marussi, Eigenvibrations of the earth observed at Trieste, Geophys. J., 6, 299-311, 1961.

Dziewonski, A. M., and F. Gilbert, Temporal variations of the seismic moment tensor and the evidence of precursive compression for two deep earthquakes, Nature, 247, 185-188, 1974.

Dziewonski, A., and M. Landisman, Great circle Rayleigh and Love wave dispersion from 100 to 900 s, Geophys. J., 19, 37-91, 1970.

Gilbert, F., Excitation of the normal modes of the earth by earthquake source, Geophys. J., 22, 223-226, 1970.

Kanamori, H., and J. J. Cipar, Focal process of the great Chilean earthquake, May 22, 1960, Phys. Earth Planet. Interiors, in press, 1975.

Kovach, R. L., and D. L. Anderson, Study of the energy of the free oscillations of the earth, J. Geophys. Res., 72, 2155-2168, 1967.

Ness, N. F., J. C. Harrison, and L. B. Slichter, Observations of the free oscillations of the earth, J. Geophys. Res., 66, 621-629, 1961.

Saito, M., Excitation of free oscillations and surface waves by a point source in a vertically heterogeneous earth, J. Geophys. Res., 72, 3689-3699, 1967.

Slichter, L. B., The fundamental free mode of the earth's inner core, Proc. Nat. Acad. Sci. U.S., 47, 186-190, 1961.

Smith, S. W., An investigation of the earth's free oscillations, Ph.D. thesis, Calif. Inst. of Technol., Pasadena, 1961.

Smith, S. W., The anelasticity of the mantle, Tectonophysics, 13, 601-622, 1972.

Wu, F. T., Lower limit of the total energy of earthquakes and partitioning of energy among seismic waves, Ph.D. thesis, Calif. Inst. of Technol., Pasadena, 1966.

(Received August 22, 1974;

revised October 29, 1974;

accepted November 14, 1974.) 